\title{
A single dose of cannabidiol reduces blood pressure in healthy volunteers in a randomized crossover study
}

\author{
Khalid A. Jadoon, ${ }^{1}$ Garry D. Tan, ${ }^{2}$ and Saoirse E. O'Sullivan' \\ 'Division of Medical Sciences \& Graduate Entry Medicine, University of Nottingham, Royal Derby Hospital Centre, Derby, \\ United Kingdom. ${ }^{2}$ The NIHR Oxford Biomedical Research Centre, Oxford Centre for Diabetes, Endocrinology \& Metabolism, \\ Churchill Hospital, Oxford University Hospitals NHS Trust, Oxford, United Kingdom.
}

BACKGROUND. Cannabidiol (CBD) is a nonpsychoactive phytocannabinoid used in multiple sclerosis and intractable epilepsies. Preclinical studies show CBD has numerous cardiovascular benefits, including a reduced blood pressure (BP) response to stress. The aim of this study was to investigate if $C B D$ reduces $B P$ in humans.

METHODS. Nine healthy male volunteers were given $\mathbf{6 0 0} \mathrm{mg}$ of CBD or placebo in a randomized, placebo-controlled, double-blind, crossover study. Cardiovascular parameters were monitored using a finometer and laser Doppler.

RESULTS. CBD reduced resting systolic BP $(-6 \mathrm{mmHg} ; P<0.05)$ and stroke volume $(-8 \mathrm{ml} ; P<$ 0.05 ), with increased heart rate (HR) and maintained cardiac output. Subjects who had taken CBD had lower BP (-5 mmHg; $P<0.05$, especially before and after stress), increased HR (+10 bpm; $P<$ $0.01)$, decreased stroke volume $(-13 \mathrm{ml} ; P<0.01)$, and a blunted forearm skin blood flow response to isometric exercise. In response to cold stress, subjects who had taken CBD had blunted BP (-6 $\mathrm{mmHg} ; P<0.01)$ and increased HR (+7 bpm; $P<0.05)$, with lower total peripheral resistance.

CONCLUSIONS. This data shows that acute administration of $C B D$ reduces resting $B P$ and the $B P$ increase to stress in humans, associated with increased HR. These hemodynamic changes should be considered for people taking CBD. Further research is required to establish whether CBD has a role in the treatment of cardiovascular disorders.

Conflict of interest: GW Pharma supplied the cannabidiol (CBD) and placebo but did not fund the study.

Submitted: March 2, 2017

Accepted: April 27, 2017

Published: June 2, 2017

\section{Reference information:}

JCI Insight. 2017;2(11):e93760. https://doi.org/10.1172/jici.

insight.93760.

\section{Introduction}

Epidemiological studies have shown a positive relationship between long-term stress and the development of cardiovascular disease (1). Factors like social isolation, low socioeconomic status, depression, stressful family and work life, and anxiety are associated with an increased risk of the development and accelerated progression of existing cardiovascular disease. Current European guidelines on the prevention of cardiovascular disease have emphasized the importance of tackling these factors (2). Mental stress induces myocardial ischaemia in patients with stable coronary artery disease, and this appears to be mediated by adrenal release of catecholamines (3)

Cannabinoids $(\mathrm{CBs})$ are compounds that bind to $\mathrm{CB}$ receptors or are structurally similar to compounds that bind to $\mathrm{CB}$ receptors. They include endogenously produced compounds (called endocannabinoids), synthetic compounds and phytocannabinoids obtained from the Cannabis sativa plant. There are over 80 known types of phytocannabinoids, the most widely studied of which is $\Delta^{9}$ tetrahydrocannabinol $\left(\Delta^{9}-\mathrm{THC}\right.$ or THC), which is responsible for the psychoactive properties of cannabis (4). The other major phytocannabinoid is cannabidiol (CBD), which does not have psychoactive properties. CBD is currently the focus of much research due to its potential in a number of therapeutic areas, as it has been shown to have antiinflammatory, anticonvulsant, antioxidant, anxiolytic, antinausea, and antipsychotic properties (5). A number of preclinical studies have also shown beneficial effects of CBD in a range of disorders of the cardiovascular system (6). A CBD/THC combination (Sativex/Nabiximols, GW Pharmaceuticals) is licensed for the treatment of spasticity in multiple sclerosis, and CBD alone (Epidiolex, GW Pharmaceuticals) has entered an expanded access program in children with intractable epilepsies (Dravet 

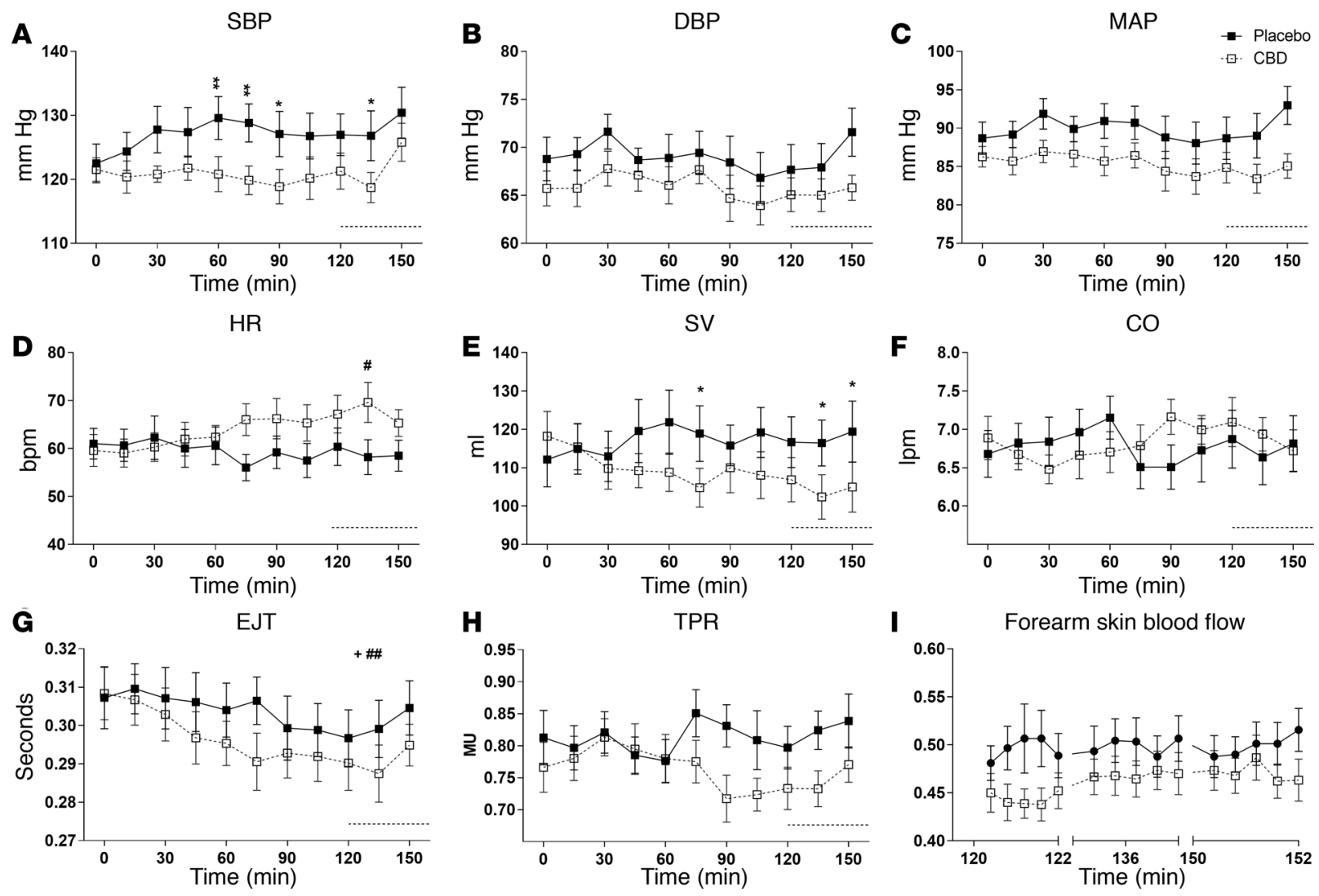

Figure 1. Changes in resting cardiovascular parameters after a single dose (600 $\mathrm{mg})$ of cannabidiol (CBD) in healthy volunteers ( $n=9)$. The effects of placebo (closed square) and CBD (open square) on systolic blood pressure (SBP) (A), diastolic blood pressure (DBP) (B), mean arterial blood pressure (MAP) (C), heart rate (HR) (D), stroke volume (SV) (E), cardiac output (CO) (F), ejection time (EJT) (G), total peripheral resistance (TPR) (H), and forearm blood flow (I), measured continuously over 2 hours after drug ingestion, except for forearm blood flow. Forearm blood was measured over a time period of 2 minutes just before the start and in between the stress tests. Dotted line denotes baseline values between the stress tests. Repeated measures 2-way ANOVA; mean $\pm \mathrm{SEM}\left({ }^{*} / I^{+} /{ }^{\#} P<0.05,{ }^{* *} /{ }^{++} /{ }^{\#} P<0.01\right.$ using Bonferroni's post-hoc analysis; + and \# represent significant change in any parameter over time seen with placebo and $\mathrm{CBD}$, respectively; denotes overall significant difference between 2 treatments).

syndrome and Lennox-Gastaut syndrome). Epidiolex has also received orphan designation status for the treatment of neonatal hypoxia-ischaemic encephalopathy.

CBD has multiple desirable effects on the cardiovascular system. It attenuates high glucoseinduced proinflammatory changes in human coronary artery endothelial cells (7) and myocardial dysfunction associated with animal models of diabetes (8), and it preserves endothelial integrity in diabetic retinal microvasculature (9). In vivo administration of CBD before cardiac ischemia and reperfusion also reduces ventricular arrhythmias and infarct size. $C B D$ also causes both acute and time-dependent vasorelaxation in isolated arteries in rats and humans (10-12). There is also evidence from animal studies that CBD modulates the cardiovascular response to stress. Resstel and colleagues (13) showed in rats that i.p. injection of CBD (10 and $20 \mathrm{mg} / \mathrm{kg},-30 \mathrm{~min})$ reduced restraint stress-induced cardiovascular response and behavior. Both these effects were blocked by preadministration of WAY 100635 $(0.1 \mathrm{mg} / \mathrm{kg})$, a 5 -hydroxytryptamine $1 \mathrm{~A}\left(5 \mathrm{HT}_{1 \mathrm{~A}}\right)$ antagonist. These effects appear to be mediated centrally and involve the bed nucleus of the stria terminalis (BNST), a limbic structure that modulates neuroendocrine responses to acute stress (14).

Our recent systematic review showed us that there are no dedicated studies in humans to date, to our knowledge, looking at the effect of CBD on either resting cardiovascular measurement or on the responses to stress, with continuous monitoring of $\mathrm{CV}$ parameters (15). Therefore, the aim of the present study was to investigate whether $\mathrm{CBD}$ decreases the cardiovascular response to stress after the administration of a single dose of $\mathrm{CBD}(600 \mathrm{mg})$ in healthy volunteers, with the hypothesis that blood pressure would be reduced by CBD. Noninvasive cardiovascular measurements were used along with stress tests in the form of mental arithmetic, isometric exercise, and the cold pressor test. 
Mental arithmetic

\begin{tabular}{|c|c|c|c|c|c|c|c|c|c|c|c|c|}
\hline \multirow[b]{3}{*}{ subject } & \multicolumn{6}{|c|}{ Systolic blood pressure } & \multicolumn{6}{|c|}{ Diastolic blood pressure } \\
\hline & \multicolumn{2}{|c|}{ Baseline } & \multicolumn{2}{|c|}{ Peak (25 s) } & \multicolumn{2}{|c|}{ Recovery } & \multicolumn{2}{|c|}{ Baseline } & \multicolumn{2}{|c|}{ Peak (25 s) } & \multicolumn{2}{|c|}{ Recovery } \\
\hline & Placebo & CBD & Placebo & CBD & Placebo & CBD & Placebo & CBD & Placebo & CBD & Placebo & CBD \\
\hline 2 & 106 & 115 & 110 & 115 & 114 & 113 & 52 & 61 & 58 & 65 & 57 & 62 \\
\hline 3 & 130 & 138 & 134 & 141 & 122 & 121 & 66 & 70 & 71 & 77 & 62 & 62 \\
\hline 5 & 124 & 123 & 126 & 124 & 121 & 115 & 76 & 67 & 70 & 67 & 74 & 64 \\
\hline 6 & 131 & 114 & 132 & 125 & 127 & 112 & 64 & 59 & 77 & 71 & 68 & 58 \\
\hline 7 & 140 & 130 & 147 & 138 & 141 & 131 & 71 & 61 & 79 & 71 & 72 & 60 \\
\hline 8 & 125 & 126 & 144 & 126 & 125 & 125 & 72 & 68 & 85 & 66 & 71 & 67 \\
\hline
\end{tabular}

\section{Isometric exercise}

\begin{tabular}{|c|c|c|c|c|c|c|c|c|c|c|c|c|}
\hline \multirow[b]{2}{*}{ subject } & \multicolumn{6}{|c|}{ Systolic blood pressure } & \multicolumn{6}{|c|}{ Diastolic blood pressure } \\
\hline & Placebo & CBD & Placebo & CBD & Placebo & CBD & Placebo & CBD & Placebo & CBD & Placebo & CBD \\
\hline 2 & 107 & 112 & 138 & 137 & 120 & 118 & 53 & 62 & 76 & 87 & 57 & 64 \\
\hline 3 & 128 & 121 & 154 & 150 & 134 & 121 & 66 & 63 & 92 & 87 & 67 & 63 \\
\hline 5 & 118 & 119 & 135 & 126 & 123 & 122 & 74 & 67 & 89 & 70 & 75 & 68 \\
\hline 6 & 133 & 114 & 131 & 149 & 135 & 123 & 72 & 60 & 65 & 83 & 68 & 66 \\
\hline 7 & 142 & 131 & 136 & 164 & 137 & 139 & 69 & 64 & 84 & 90 & 75 & 67 \\
\hline 8 & 131 & 121 & 144 & 134 & 137 & 121 & 77 & 68 & 94 & 76 & 78 & 64 \\
\hline
\end{tabular}

\section{Cold pressor test}

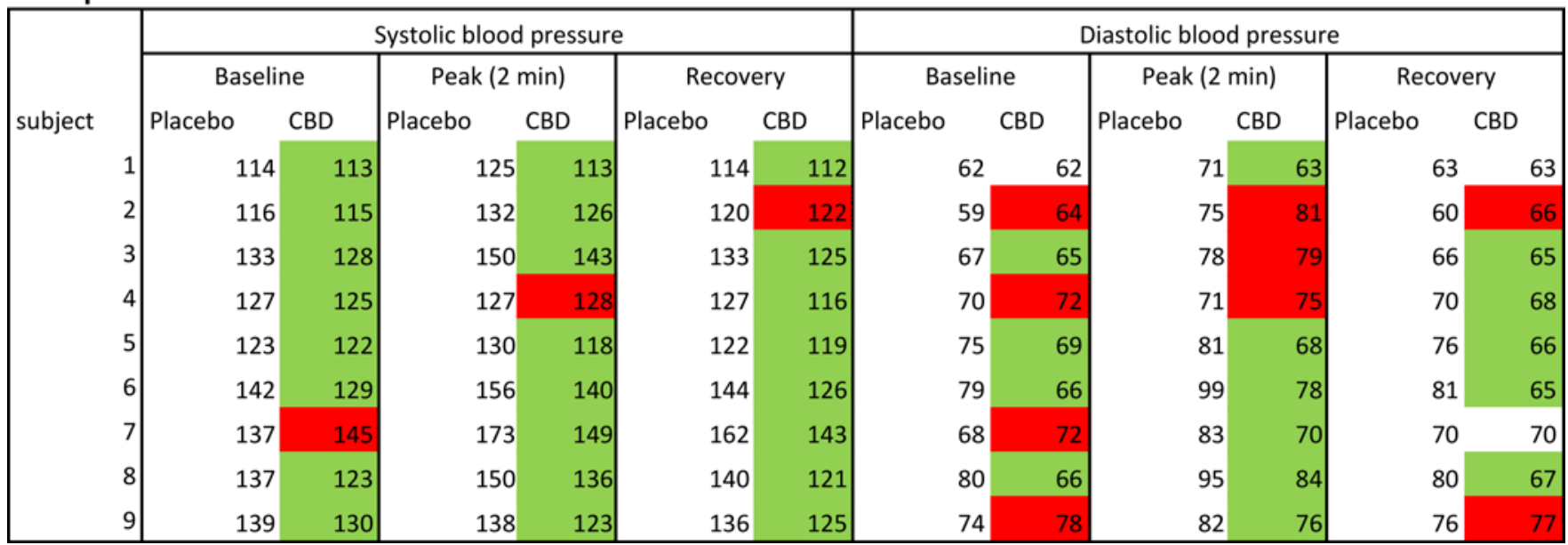

Figure 2. Individual systolic and diastolic blood pressure responses to all stress tests after a single dose (600 mg) of cannabidiol (CBD) or placebo in healthy volunteers $(\boldsymbol{n}=\mathbf{9})$. Green color coding shows subjectS who had a reduced (compared with placebo) blood pressure response to stress after taking CBD, and red color coding shows an increased blood pressure response to stress after taking CBD.

\section{Results}

Ten male subjects were recruited, but 1 withdrew for personal reasons. The mean age, weight, and height of the volunteers were $23.7 \pm 3.2$ years, $77.5 \pm 6.4 \mathrm{~kg}$, and $178.6 \pm 4.5 \mathrm{~cm}$ (mean $\pm \mathrm{SD}$ ).

Effect of $C B D$ on resting cardiovascular parameters. $\mathrm{CBD}$ treatment reduced resting systolic blood pressure 

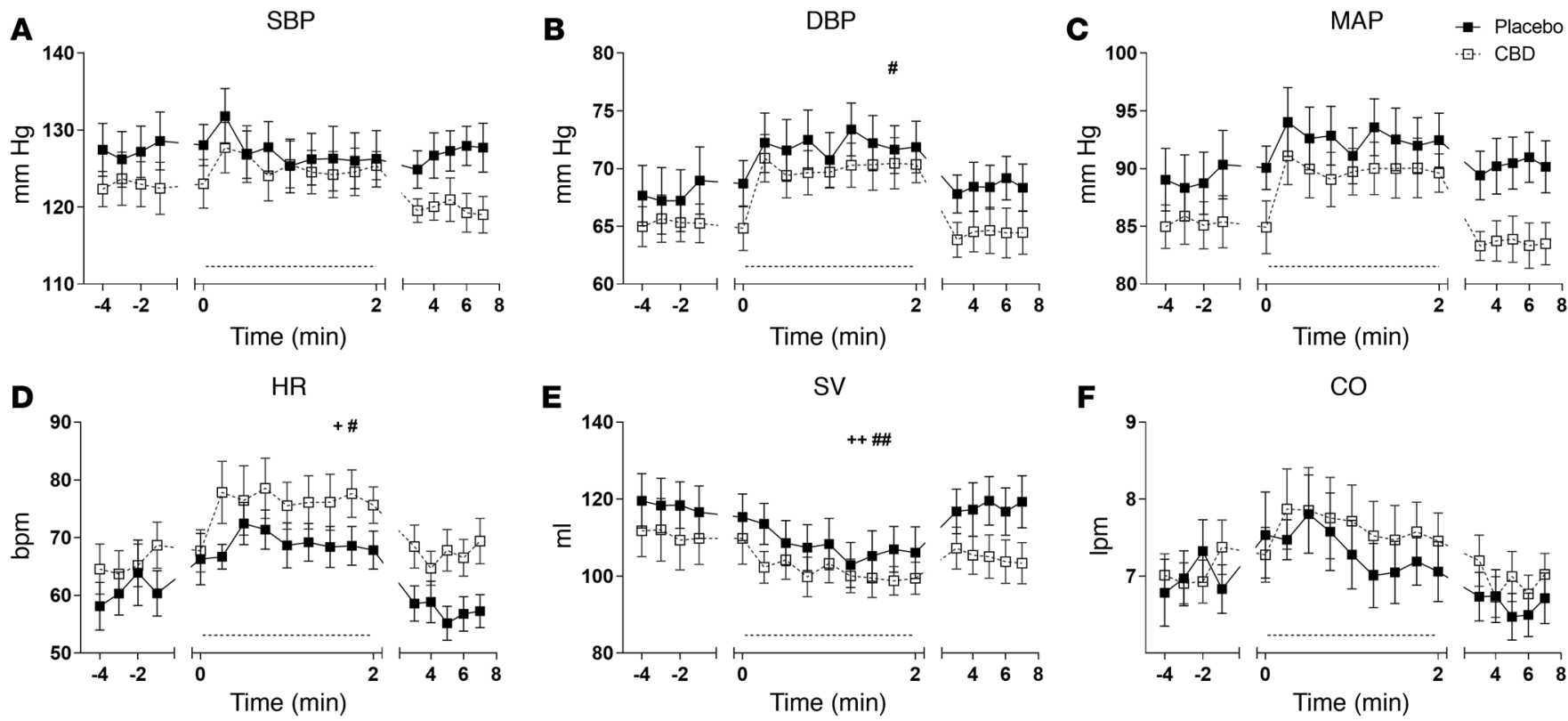

$\mathbf{F}$

$\mathrm{CO}$
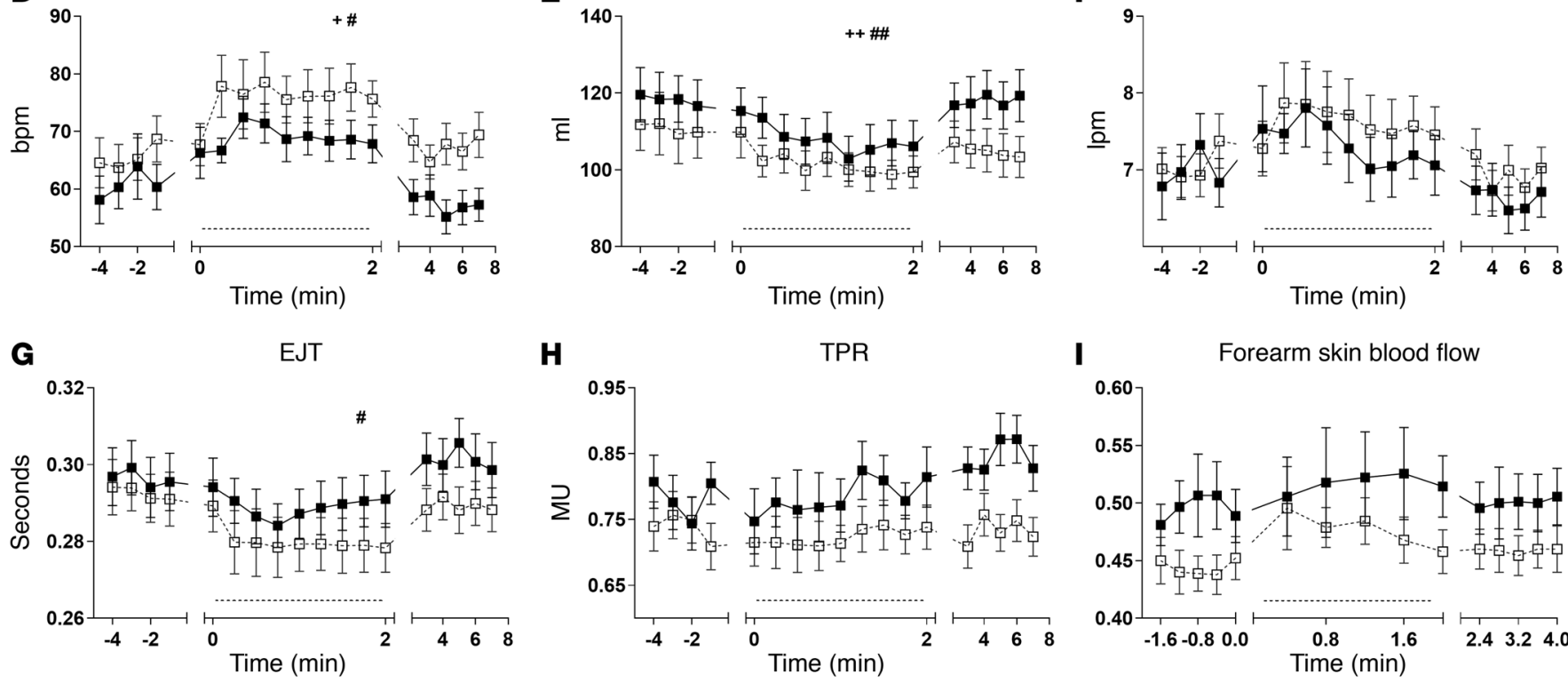

Figure 3. Cardiovascular response to mental stress after a single dose $(\mathbf{6 0 0} \mathbf{~ m g})$ of cannabidiol (CBD) in healthy volunteers $(\boldsymbol{n}=\mathbf{9})$. The effects of placebo (closed square) and CBD (open square) on systolic blood pressure (SBP) (A), diastolic blood pressure (DBP) (B), mean arterial blood pressure (MAP) (C), heart rate (HR) (D), stroke volume (SV) (E), cardiac output (CO) (F), ejection time (EJT) (C), total peripheral resistance (TPR) (H), and forearm blood flow (I), measured continuously just before, during, and after mental arithmetic test (dotted line denotes stress test period), except for forearm blood flow. Measurements for forearm blood flow were made over a 2-minute window just before, during, and after the stress test. Repeated measures 2-way ANOVA; mean \pm SEM (+ and \# denote significant change in a parameter during the stress period seen with placebo and CBD, respectively). ${ }^{+} /{ }^{\#}<<0.05,{ }^{++} / \#^{\#} \mathrm{P}<0.01$.

(SBP) (mean difference $-6 \mathrm{mmHg} ; 95 \% \mathrm{CI},-1$ to $-12, P<0.05$, Figure 1A). Although there was no overall difference in diastolic blood pressure (DBP) and mean arterial blood pressure (MAP) between the 2 groups, post-hoc analysis showed that both DBP and MAP were lower with CBD treatment (Figure 1, B and C, respectively; $P<0.01)$, particularly in the latter time points $(120-160 \mathrm{~min})$.

$\mathrm{CBD}$ treatment reduced resting stroke volume (SV; mean difference $-8 \mathrm{ml} ; 95 \% \mathrm{CI},-2$ to $-14, P<0.05$, Figure 1E) and increased heart rate (HR), with significant difference in HR between CBD and placebo from an hour after ingestion of drug (Figure $1 \mathrm{D}, P<0.05)$. There was a decline in left ventricular ejection time (EJT) with both treatments $(P<0.05$; Figure $1 \mathrm{G})$, while cardiac output $(\mathrm{CO})$ (Figure $1 \mathrm{~F}$ ) remained unchanged.

There was a trend toward reduction in total peripheral resistance (TPR, Figure $1 \mathrm{H})$ with CBD in the latter half of the resting period, and a significant reduction in forearm skin blood flow before the start of the stress tests (Figure 1I; $P<0.01$ ).

Effect of CBD on cardiovascular parameters mental stress. The individual blood pressure responses of healthy volunteers to the stresses are presented in Figure 2, showing the average baseline systolic or diastolic blood pressure in the 4 minutes preceeding the stress test, the peak response during stress, and the average recovery response in the 4 minutes after the stress test.

Mental stress test. Mental stress caused a rise in $\mathrm{HR}(P<0.05$; Figure $3 \mathrm{D})$ and a decline in SV $(P<0.01$; Figure $3 \mathrm{E})$, which was seen in both the $\mathrm{CBD}$ and placebo groups. There was a rise in $\mathrm{DBP}(P<0.05$; Figure 3B) and a decline in EJT $(P<0.05$; Figure $3 G)$, seen only in those who had taken CBD. 

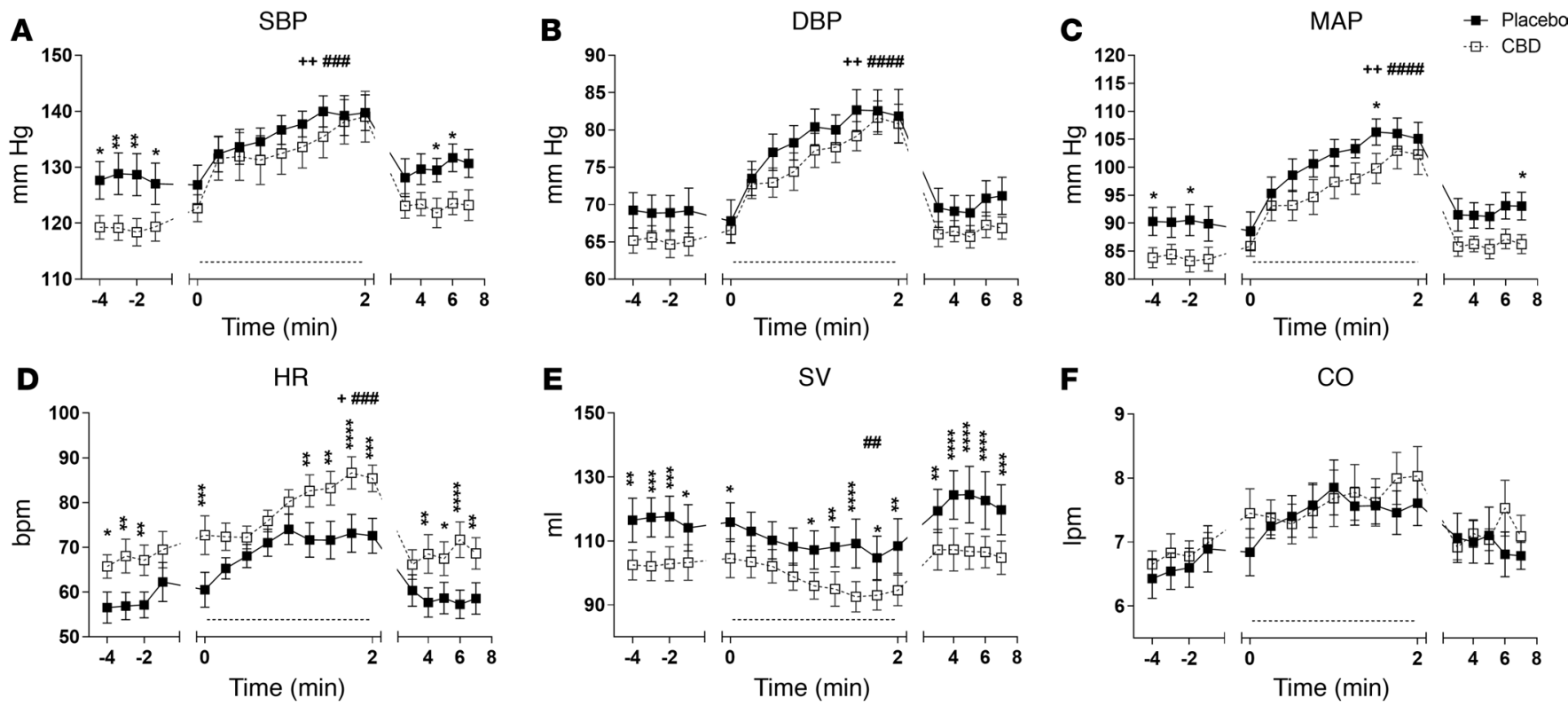

$\mathbf{F}$

$\mathrm{CO}$
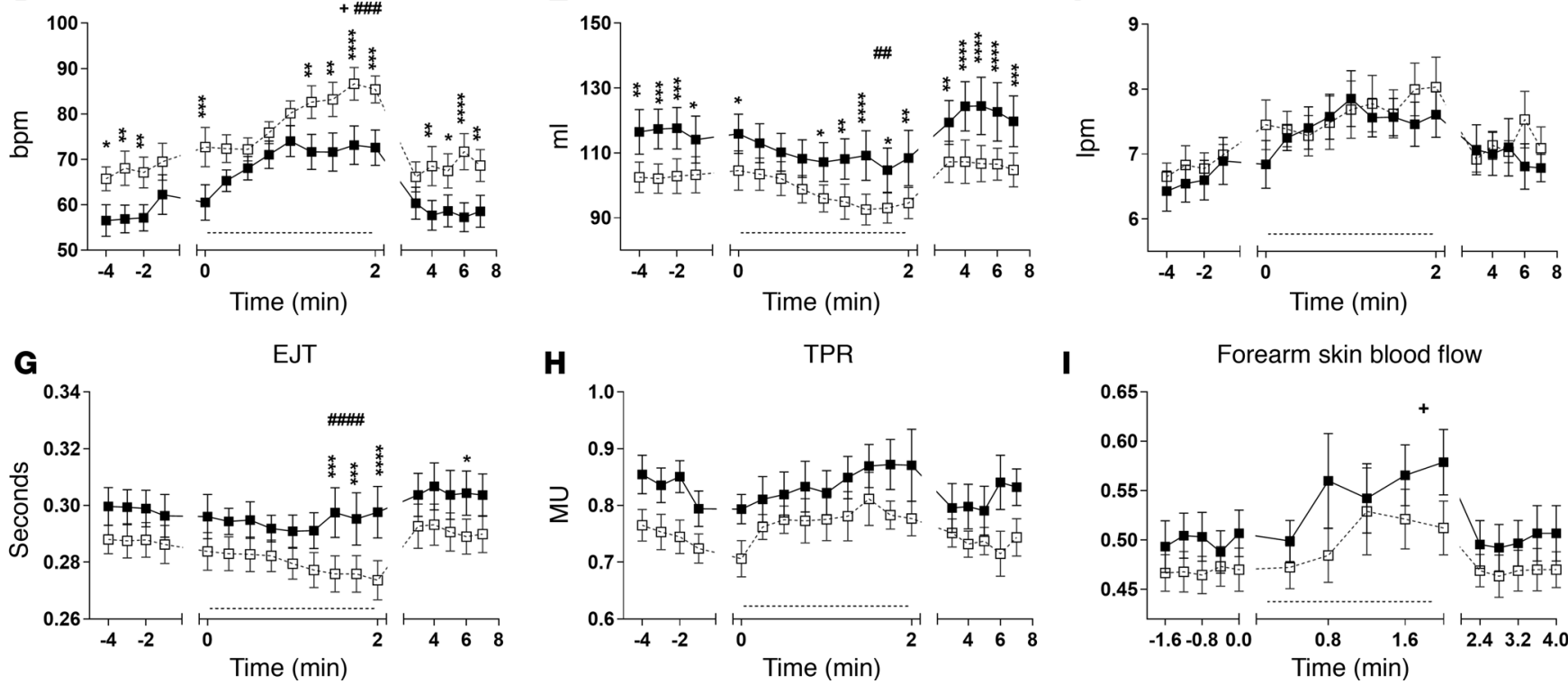

Figure 4. Cardiovascular parameters in response to exercise stress after a single dose $(\mathbf{6 0 0} \mathbf{m g})$ of cannabidiol (CBD) in healthy volunteers $(\boldsymbol{n}=\mathbf{9})$. The effects of placebo (closed square) and CBD (open square) on systolic blood pressure (SBP) (A), diastolic blood pressure (DBP) (B), mean arterial blood pressure (MAP) (C), heart rate (HR) (D), stroke volume (SV) (E), cardiac output (CO) (F), ejection time (EJT) (C), total peripheral resistance (TPR) (H), and forearm blood flow (I), measured continuously just before, during, and after isometric exercise test (dotted line denotes stress test period), except for forearm blood flow. Measurements for forearm blood flow were made over a 2-minute window just before, during, and after the stress test. Repeated measures 2-way

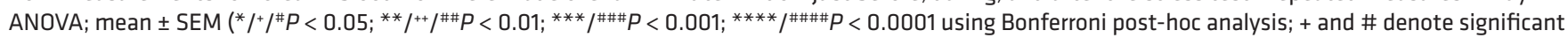
change in a parameter during the stress period seen with placebo and CBD respectively).

Although overall, there was no difference in the cardiovascular parameters between the 2 treatments, post-hoc analysis showed that SBP (Figure 3A; $P<0.05$ to $<0.0001$ ), DBP (Figure 3B; $P<0.05$ to $<0.01$ ), and MAP (Figure 3C; $P<0.05$ to $<0.0001$ ) were significantly lower in volunteers who had taken CBD, especially immediately after the stress test. Looking at the individual response to mental stress, 6 of 9 subjects had a lower SBP before or during the mental stress test, and 9 of 9 subject had a lower SBP in the recovery period after taking CBD (Figure 2). Five of 9 subjects had a lower DBP during the mental stress test, and 6 of 9 subject had a lower DBP in the recovery period after taking CBD (Figure 2).

$\mathrm{CBD}$ increased $\mathrm{HR}$ during the latter part of mental stress and in the poststress period (Figure 3D; $P<0.05$ to $<0.0001$ ), with a corresponding decline in EJT (Figure 3G; $P<0.05$ to $<0.0001$ ). Although CBD reduced SV (Figure 3E; $P<0.05$ to $<0.0001$ ), there was no difference in CO between CBD and placebo (Figure 3F).

Compared with placebo, volunteers who had taken CBD had a lower TPR throughout and especially in the post-mental stress period (Figure $3 \mathrm{H} ; P<0.05$ to $<0.0001$ ). Volunteers who had taken $\mathrm{CBD}$ also reduced forearm skin blood flow before and during the latter part of mental stress (Figure 3I; $P<$ 0.05 to $<0.01)$.

Exercise stress test. The isometric exercise stress caused a significant rise in the following parameters in both treatment groups; SBP (placebo $P<0.01$, CBD $P<0.001$; Figure 4A), DBP (placebo $P<0.01, \mathrm{CBD}$ $P<0.0001$; Figure 4B), MAP (placebo $P<0.01$, CBD $P<0.0001$; Figure 4C), and HR (placebo $P<0.05$, CBD $P<0.001 ;$ Figure 4D). 

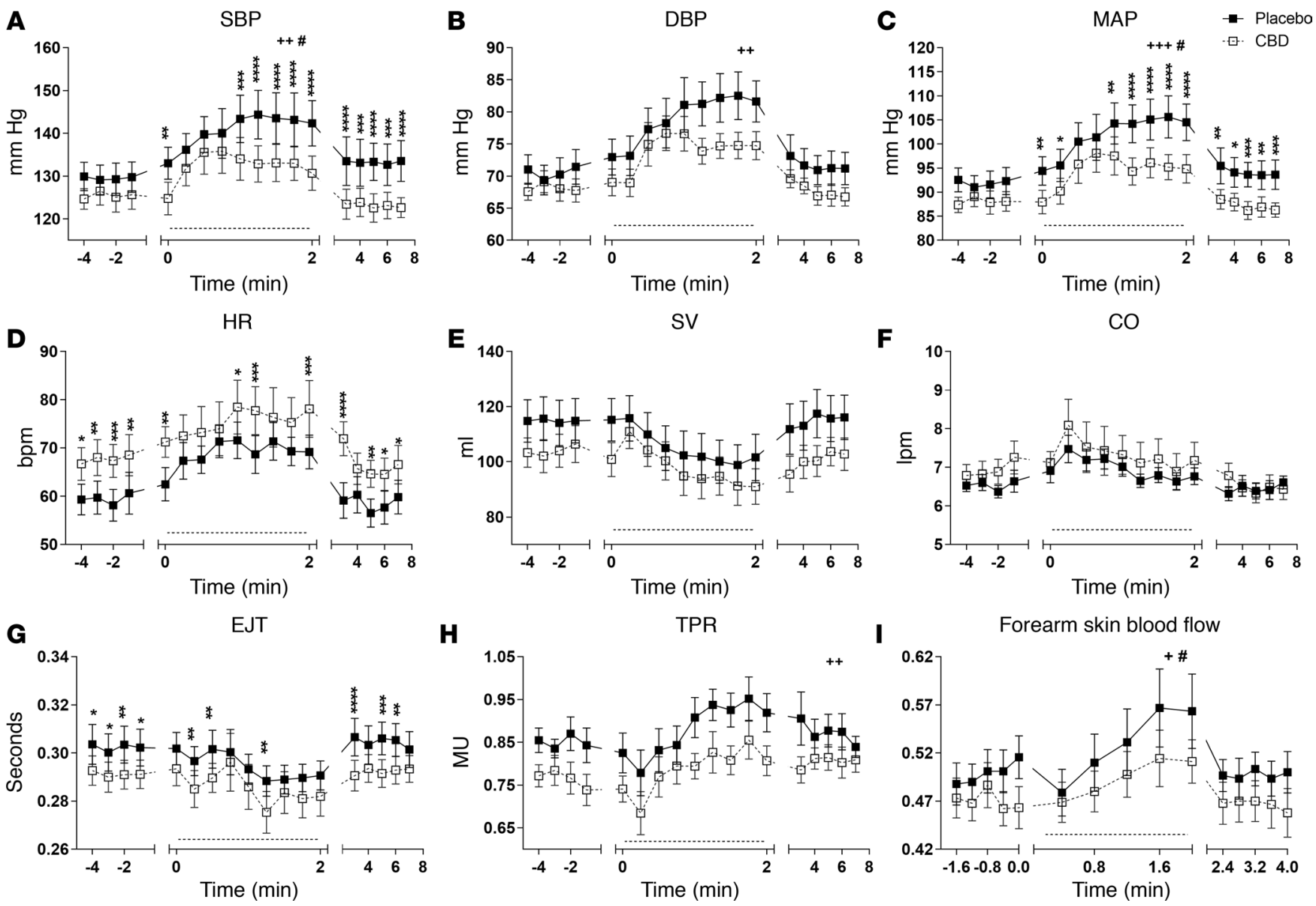

Figure 5. Cardiovascular response to cold stress after a single dose $(\mathbf{6 0 0} \mathbf{m g})$ of cannabidiol (CBD) in healthy volunteers $(\boldsymbol{n}=\mathbf{9})$. The effects of placebo (closed square) and CBD (open square) on systolic blood pressure (SBP) (A), diastolic blood pressure (DBP) (B), mean arterial blood pressure (MAP) (C), heart rate (HR) (D), stroke volume (SV) (E), cardiac output (CO) (F), ejection time (EJT) (C), total peripheral resistance (TPR) (H), and forearm blood flow (I), measured continuously just before, during, and after cold pressor test (dotted line denotes stress test period), except for forearm blood flow. Measurements for forearm blood flow were made over a 2-minute window just before, during, and after the stress test. Repeated measures 2 -way ANOVA; mean \pm SEM $\left(^{*} /{ }^{+} /^{\#} P<0.05\right.$, ${ }^{* *} I^{++} P<0.01,{ }^{* *} / I^{+++} P<0.001,{ }^{* * *} P<0.0001$ using Bonferroni post-hoc analysis; + and \# denote significant change in a parameter during the stress period seen with placebo and CBD, respectively).

Overall, CBD treatment reduced SBP (mean difference $-5 \mathrm{mmHg} ; 95 \% \mathrm{CI},-1$ to $-10, P<0.05$, Figure $4 \mathrm{~A}$ ) and MAP (mean difference $-5 \mathrm{mmHg}, 95 \% \mathrm{CI},-2$ to $-9, P<0.05$, Figure $4 \mathrm{C}$ ) during the exercise stress. Looking at the individual response to exercise stress, 6 of 9 subjects had a lower SBP during isometric exercise and 8 of 9 subjects had a lower SBP in the recovery period after taking CBD (Figure 2). Five of 9 subjects had a lower DBP during exercise, and 8 of 9 subject had a lower DBP in the recovery period after taking CBD (Figure 2).

Subjects who had taken CBD had increased HR (mean difference $10 \mathrm{bpm} ; 95 \% \mathrm{CI}, 5-14, P<0.01$, Figure 4D) and decreased SV (mean difference $-13 \mathrm{ml}$; 95\% CI, -4 to $-22, P<0.01$, Figure $4 \mathrm{E}$ ) and EJT (mean difference $-0.01 \mathrm{sec}, 95 \% \mathrm{CI},-0.001$ to $-0.03, P<0.05$, Figure $4 \mathrm{G}$ ) during the exercise stress. There was no difference in $\mathrm{CO}$ during the exercise stress (Figure $4 \mathrm{~F}$ ).

A rise in forearm cutaneous blood flow in response to exercise (as would be expected) was only seen in volunteers who had taken placebo $(P<0.05$; Figure $4 \mathrm{I})$. Post-hoc analysis showed significantly lower forearm skin blood flow in those who had taken CBD (Figure 4I; $P<0.001$ to $<0.0001$ ), during the early and latter parts of the stress test. This was associated with reduced TPR (Figure $4 \mathrm{H} ; P<0.05$ to $<0.001$ ) before, after, and in the latter half of exercise stress.

Cold stress test. The cold pressor test caused a rise in SBP (placebo $P<0.01, \mathrm{CBD} P<0.05$; Figure 5A) and MAP (placebo $P<0.001, \mathrm{CBD} P<0.05$; Figure $5 \mathrm{C}$ ) in both groups and a rise in DBP only with placebo $(P<0.01$; Figure $5 \mathrm{~B})$. An equal rise in $\mathrm{SBP}$ and MAP was seen with both $\mathrm{CBD}$ and placebo in the first half of this stress test. However, while the blood pressure (SBP and MAP) continued to rise in the placebo group, it plateaued in volunteers who had taken $\mathrm{CBD}$, and therefore both SBP and MAP were significantly 
lower in volunteers after CBD (mean difference $-8 \mathrm{mmHg}[95 \% \mathrm{CI},-4$ to $-12, P<0.01]$ and $-6 \mathrm{mmHg}$ [95\% CI, -2 to $-11, P<0.01]$ ). Post-hoc analysis showed that DBP was also significantly lower in those who had taken CBD in the latter half of the stress period (Figure $5 \mathrm{~B} ; P<0.001$ ).

Looking at the individual response to the cold pressor test, 8 of 9 subjects had a lower SBP during the cold stress and in the recovery period after taking CBD (Figure 2). Six of 9 subjects had a lower DBP during the cold pressor, and 7 of 9 subject had a lower DBP in the recovery period after taking CBD (Figure 2).

As before, HR was higher in volunteers who had taken CBD (mean difference 7 bpm; 95\% CI: 2-13; $P<0.05$; Figure 5D), and EJT was lower (mean difference $-0.01 \mathrm{sec} ; 95 \% \mathrm{CI}$ : 0 to $-0.02 ; P<0.05$; Figure $5 \mathrm{G})$. Sidak post-hoc analysis showed that SV was significantly lower with CBD throughout (Figure 5E; $P<$ 0.05 to $<0.0001$ ), but there was no decline in cardiac output (Figure $5 \mathrm{~F}$ ).

The cold pressor test caused a significant rise in TPR (as expected) in the placebo group only (Figure $5 \mathrm{H} ; P<0.01$ ) and rise in forearm skin blood flow with both $\mathrm{CBD}$ and placebo (Figure 5I; $P<0.05$ ). The overall trend was for lower TPR and forearm skin blood flow in those who had taken CBD, with post-hoc analysis showing a reduction in both just before and in the latter half of cold stress (Figure $5 \mathrm{H}[P<0.05$ to $<0.0001]$ and Figure 5I $[P<0.001])$.

\section{Discussion}

Based on preclinical evidence, the aim of this study was to test the hypothesis that CBD would reduce the cardiovascular response to stress in healthy volunteers. We found that resting blood pressure was lower after subjects had taken $\mathrm{CBD}$ and that $\mathrm{CBD}$ blunted the blood pressure response to stress, particularly in the pre- and poststress periods. Post-hoc analysis showed an overall trend of lower SBP, MAP, DBP, SV, TPR, forearm skin blood flow, and left ventricular EJT and a higher HR in subjects who had taken CBD. These hemodynamic changes should be considered for people taking CBD and suggest that further research is warranted to establish whether $\mathrm{CBD}$ has any role in the treatment of cardiovascular disorders.

We have shown for the first time that to our knowledge that, in humans, acute administration of CBD reduces resting blood pressure, with a lower stroke volume and a higher heart rate. This response may be secondary to the known anxiolytic properties of CBD (16) and may account for the lack of anticipatory rise in blood pressure seen with placebo. These findings are in contrast to previous studies in humans, where CBD at the same dose did not affect baseline cardiovascular parameters (17-19), although changes in the cardiovascular system were not the primary outcome of these studies. In the present study, CV parameters were measured continuously, while in previous studies, monitoring for SBP, DBP, and HR were performed manually at only 1, 2, or 3 hours after drug delivery. Additionally, our subjects were cannabis naive, while the subjects of other studies had used cannabis in the past. Since tolerance may develop to the hemodynamic response to $\mathrm{CBs}$ in humans, this may explain the differences between studies.

THC, the major psychoactive component of cannabis, is known to cause tachycardia and orthostatic hypotension in humans (20), a hemodynamic response similar to that observed to CBD in the present study. THC is a partial agonist at both $\mathrm{CB}_{1}$ and $\mathrm{CB}_{2}$ receptors (21), and the effects of THC on heart rate are mediated through $\mathrm{CB}_{1}$ receptors (20). $\mathrm{CBD}$ does not bind with any great affinity to $\mathrm{CB}_{1}$, but it can interact indirectly by augmenting $\mathrm{CB}_{1}$ receptors' constitutional activity or endocannabinoid tone, the so called indirect agonism (22). We recently showed that CBD also causes endothelium-dependent vasorelaxation in isolated human mesenteric arteries through $\mathrm{CB}_{1}$ activation (11). Therefore, it is possible that the changes in hemodynamics brought about by $\mathrm{CBD}$ are mediated through $\mathrm{CB}_{1}$.

$\mathrm{CBD}$ may cause sympathoinhibition (through $\mathrm{CB}_{1}$ or some other mechanism), thereby preventing an increase in blood pressure and cardiac output, causing a compensatory rise in heart rate to maintain cardiac output. Indeed, the changes in SBP preceded any changes in HR. Another possibility is that CBD inhibits cardiac vagal tone, thereby increasing heart rate (despite any potential sympathoinhibition). A recent study in male Sprague-Dawley rats showed that GPR18 activation in the rostral ventrolateral medulla (RVLM) by abnormal CBD (Abn-CBD) resulted in reduced blood pressure and increased heart rate (23) (similar to that observed in the present study). The same study showed that pretreatment with atropine and propranolol fully abrogated the HR response, suggesting a role for the autonomic nervous system. CBD is a weak partial agonist at GPR18 (24).

Effect of $C B D$ on cardiovascular parameters in response to mental stress. Mental arithmetic has been shown to cause a rise in MAP and muscle sympathetic nerve activity (MSNA) (25) and vasodilatation in forearm skeletal muscle (26). In our study, none of the cardiovascular parameters other than HR, 
DBP, and SV were affected, suggesting that the level of stress to this test was minimal. This could be because of the added visual stimulus of a computer screen, which would have helped volunteers perform the task. Overall, there was trend for lower SBP, DBP, MAP, SV, TPR, and forearm skin blood flow in subjects who had taken CBD, particularly in the pre- and post-stress test periods. Like resting cardiovascular parameters, these changes may indicate anxiolytic effects of CBD and/or generalized sympathoinhibition.

Effect of $C B D$ on cardiovascular parameters in response to exercise stress. Isometric exercise produces a pressor response, via sympathoexcitation, originating in the contracting muscle and relayed to the RVLM via the nucleus of solitary tract. The end result is a rise in heart rate and cardiac output and vasoconstriction in nonexercising organs (27-29). There is increased skeletal muscle blood flow in the nonexercising limb, which is sensitive to atropine and propranolol (30). A similar response was seen in our study, where isometric exercise caused a significant rise in SBP, DBP, MAP, and HR and an increase in forearm blood flow, although this was significant in the placebo group only. Subjects who had taken CBD had reduced blood pressure during the exercise stress test, and this was most pronounced in the pre- and posttest period. Before the exercise stress, HR was higher and SV lower in volunteers when they had taken CBD, and this trend continued throughout exercise stress and in the poststress period. There was also a significant reduction in EJT with CBD, which represents a reciprocal change to increased HR. The rise in cutaneous blood flow was only seen with placebo and not with $C B D$, possibly suggesting reduced $\beta_{2}$ adrenergic-mediated vasodilatation, which could be a result of general sympathoinhibition or a specific effect at the $\beta_{2}$ adrenoceptors. The tissue distribution of $\beta_{2}$ adrenoceptors and $\mathrm{CB}_{1}$ receptors overlaps in many tissues, including in the cardiovascular system (31). At the cellular level, a complex physical and functional interaction between these 2 receptors has been demonstrated; there is evidence of cointernalization of $\beta_{2}$ adrenoceptors with $\mathrm{CB}_{1}$ receptors, leading to desensitisation of $\beta_{2}$ adrenoceptors (31).

Effect of $C B D$ on cardiovascular parameters in response to cold stress. Cold stress causes intense sympathoexcitation, producing a tachycardic and pressor response, and an increase in MSNA (32, 33). The pressor response is due to an initial rise in CO, in response to increased HR and a later increase in MSNA, causing vasoconstriction. Both MAP and TPR show a linear correlation with MSNA during cold stress (34). In our study, cold stress produced a pressor response in both groups, but, interestingly, while SBP and MAP continued to rise with placebo throughout the test period, the pressor response to cold was blunted in subjects who had taken CBD, and SBP and MAP were significantly lower. In keeping with this, TPR was lower with CBD than placebo, suggesting a possible inhibition of sympathetic outflow. This could also be due to analgesic properties of CBD (35), reducing cold stress and therefore minimizing the sympathetic response (also explaining why the cold pressor test was affected more by CBD than the exercise test). In the animal study of Resstel and colleagues (13), the authors suggested that the modulation of cardiovascular response was most likely secondary to attenuation of emotional response to stress. However, given our findings that $\mathrm{CBD}$ produced similar changes in cardiovascular parameters - though to a variable degree — during rest and stress, this may indicate that CBD also has direct cardiovascular effects.

Safety and tolerance. CBD was well tolerated, and there were no adverse events on the day of stress tests. None of the subjects reported any adverse events over the following week.

Conclusion. Our data show that a single dose of CBD reduces resting blood pressure and the blood pressure response to stress, particularly cold stress, and especially in the post-test periods. This may reflect the anxiolytic and analgesic effects of $\mathrm{CBD}$, as well as any potential direct cardiovascular effects. CBD also affected cardiac parameters but without affecting cardiac output. Giving the increasing use of CBD as a medicinal product, these hemodynamic changes should be considered for people taking CBD. Further research is also required to establish whether $\mathrm{CBD}$ has any role in the treatment of cardiovascular disorders such as a hypertension.

\section{Methods}

Study design. The study was a randomized, crossover design with each subject given CBD (BN: K12067A) or placebo (both gifts from GW Pharmaceuticals) in a capsule in a double-blind fashion, with a minimum time interval of at least 48 hours (range 3-16 days), taking place at the Division of Medical Sciences, School of Medicine, Royal Derby Hospital. Allocation was decided by a coin toss, and block randomization was employed by S.E. O'Sullivan, who assigned participants. K.A. Jadoon carried out all study visits, and data analysis was blinded. 
During an initial visit, subjects were familiarized with the stress tests and with noninvasive cardiovascular (CVS) monitoring, and an electrocardiogram (ECG) was done to rule out any preexisting cardiac conditions. Subjects were advised to fast overnight, to avoid beverages containing caffeine or alcohol, and to avoid strenuous exercise for 24 hours before each of the 2 study visits. Two hours after CBD/placebo was administered, subjects performed various stress tests (36). Noninvasive cardiovascular monitoring using Finometer and laser Doppler flowmetry was carried out during the 2 hours to assess changes in baseline parameters and during the stress test periods.

Visit days. Upon arrival, subjects were rested for 10-15 minutes, and their baseline blood pressure and heart rate were recorded using a digital blood pressure (BP) monitor. Participants were given a standardized breakfast, and 15 minutes later, they were given either oral CBD (600 mg) or placebo in a double-blind fashion. This is a dose known to cause anxiolytic effects in humans and is comparable with what is used clinically $(19,37-39)$. Study medication consisted of capsules containing either $100 \mathrm{mg}$ of CBD or excipients, which were a gift from GW Pharmaceuticals. There was no difference between the 2 formulations in color, taste, or smell.

Two hours afterward, subjects were asked to perform the stress tests (36). Timing of the tests was chosen to coincide with peak plasma levels for CBD (18). All the experiments were performed in a sitting position under ambient temperature conditions. Maximum voluntary contraction for the isometric hand grip test was assessed for each subject prior to administering study medication.

After administration of CBD or placebo, subjects remained seated, either doing nothing, reading, or using a computer. During this time, subjects were connected to a calibrated Finometer (Finapres Medical Systems), which uses a finger-clamp method to detect beat-to-beat changes in digital arterial diameter using an infrared photoplethysmograph (40). The Finometer gives a continuous signal of beat-to-beat changes in blood pressure and blood flow, and it uses this signal to derive other parameters, including systolic, diastolic, and mean blood pressure; interbeat interval; heart rate and left ventricular ejection time; stroke volume; cardiac output; and systemic peripheral resistance. Baseline cardiovascular data was recorded for 2 hours following administration of CBD or placebo. Forearm blood flow was measured using a calibrated laser Doppler flowmeter (Perimed) (41). For each recording, 5 images of microcirculation were taken, over an area $19 \mathrm{~mm} \times 19 \mathrm{~mm}$, using the upper third of the left forearm under high resolution. After 2 hours, subjects underwent the cardiovascular stress tests in the following order: mental arithmetic, isometric exercise, and cold pressor test.

The mental arithmetic test consisted of calculating a sum every 2 second for 2 minutes. Subjects were seated in front of a computer screen, and a PowerPoint presentation delivered a slide with a simple mathematical sum of a 3-digit number minus a smaller number (e.g., 317 - 9, 212 - 11, 185 - 7) every 2 seconds; the subject had to give the answer verbally. In the isometric exercise stress test, using a dynamometer, handgrip was maintained at $30 \%$ of maximum voluntary contraction (MVC) for $2 \mathrm{~min}$. For the cold pressor test, subjects immersed their left foot (up to ankle) in ice slush (temperature $4^{\circ} \mathrm{C}-6^{\circ} \mathrm{C}$ ) for 2 minutes. Cardiovascular parameters were measured continuously using the Finometer, while skin blood flow measurements were taken just before, during, and 5 minutes after each test. Each stress test lasted for 2 minutes, and there was a recovery period of at least 10 minutes.

Statistics. Data were analyzed using repeated measures ANOVA to determine the effect of treatment and time on different variables using GraphPad PRISM version 6.02. Level of significance was set at $\alpha=0.05$ and values presented as mean \pm SEM. Sidak's post-hoc test was used to see treatment affect at various time points. Data were not unblinded until after statistical analysis.

Study approval. Ten healthy young male volunteers, mean age 24 years (range 19-29), with no underlying cardiovascular or metabolic disorders, were recruited for this study, which was approved by the University of Nottingham Faculty of Medicine Ethics Committee (study reference E18102012). Written informed consent was obtained according to the Declaration of Helsinki. Exclusion criteria included any significant cardiovascular or metabolic disorder or use of any medication. All the volunteers were nonsmokers and had taken no prescribed or over-the-counter medication within a week prior to randomization. No volunteers had ever used cannabis.

\section{Author contributions}

KAJ helped with study design, researched data, wrote the manuscript, and reviewed/edited the manuscript. GDT reviewed/edited the manuscript. SEO was involved in study design and reviewed/edited the manuscript. 


\section{Acknowledgments}

GT is supported by the NIHR Oxford Biomedical Research Centre Programme. The views expressed are those of the author and not necessarily those of the NHS, the NIHR, or the Department of Health.

Address correspondence to: Saoirse E. O'Sullivan, Division of Medical Sciences \& Graduate Entry Medicine, University of Nottingham, Royal Derby Hospital Centre, Uttoxeter Road, Derby DE22 3DT, United Kingdom. Phone: 44.1332.724701; E-mail:mbzso@nottingham.ac.uk.

1. Figueredo VM. The time has come for physicians to take notice: the impact of psychosocial stressors on the heart. Am JMed. 2009;122(8):704-712.

2. Perk J, et al. European Guidelines on cardiovascular disease prevention in clinical practice (version 2012). The Fifth Joint Task Force of the European Society of Cardiology and Other Societies on Cardiovascular Disease Prevention in Clinical Practice (constituted by representatives of nine societies and by invited experts). Eur Heart J. 2012;33(13):1635-1701.

3. Goldberg AD, et al. Ischemic, hemodynamic, and neurohormonal responses to mental and exercise stress. Experience from the Psychophysiological Investigations of Myocardial Ischemia Study (PIMI). Circulation. 1996;94(10):2402-2409.

4. Costa B. On the pharmacological properties of Delta9-tetrahydrocannabinol (THC). Chem Biodivers. 2007;4(8):1664-1677.

5. Mechoulam R, Parker LA, Gallily R. Cannabidiol: an overview of some pharmacological aspects. J Clin Pharmacol. 2002;42(11 Suppl):11S-19S.

6. Stanley CP, Hind WH, O'Sullivan SE. Is the cardiovascular system a therapeutic target for cannabidiol? Br J Clin Pharmacol. 2013;75(2):313-322

7. Rajesh M, et al. Cannabidiol attenuates high glucose-induced endothelial cell inflammatory response and barrier disruption. Am J Physiol Heart Circ Physiol. 2007;293(1):H610-H619.

8. Rajesh M, et al. Cannabidiol attenuates cardiac dysfunction, oxidative stress, fibrosis, and inflammatory and cell death signaling pathways in diabetic cardiomyopathy. J Am Coll Cardiol. 2010;56(25):2115-2125.

9. El-Remessy AB, Al-Shabrawey M, Khalifa Y, Tsai NT, Caldwell RB, Liou GI. Neuroprotective and blood-retinal barrier-preserving effects of cannabidiol in experimental diabetes. Am J Pathol. 2006;168(1):235-244.

10. O'Sullivan SE, Sun Y, Bennett AJ, Randall MD, Kendall DA. Time-dependent vascular actions of cannabidiol in the rat aorta. Eur J Pharmacol. 2009;612(1-3):61-68.

11. Stanley CP, Hind WH, Tufarelli C, O'Sullivan SE. Cannabidiol causes endothelium-dependent vasorelaxation of human mesenteric arteries via CB1 activation. Cardiovasc Res. 2015;107(4):568-578.

12. Walsh SK, Hepburn CY, Kane KA, Wainwright CL. Acute administration of cannabidiol in vivo suppresses ischaemia-induced cardiac arrhythmias and reduces infarct size when given at reperfusion. Br J Pharmacol. 2010;160(5):1234-1242.

13. Resstel LB, Tavares RF, Lisboa SF, Joca SR, Corrêa FM, Guimarães FS. 5-HT1A receptors are involved in the cannabidiol-induced attenuation of behavioural and cardiovascular responses to acute restraint stress in rats. Br J Pharmacol. 2009;156(1):181-188.

14. Choi DC, Furay AR, Evanson NK, Ostrander MM, Ulrich-Lai YM, Herman JP. Bed nucleus of the stria terminalis subregions differentially regulate hypothalamic-pituitary-adrenal axis activity: implications for the integration of limbic inputs. $J$ Neurosci. 2007;27(8):2025-2034.

15. Sultan SR, Millar SA, England TJ, O'Sullivan SE. A Systematic Review and Meta-Analysis of the Haemodynamic Effects of Cannabidiol. Front Pharmacol. 2017;8:81.

16. Zuardi AW, Shirakawa I, Finkelfarb E, Karniol IG. Action of cannabidiol on the anxiety and other effects produced by delta 9-THC in normal subjects. Psychopharmacology (Berl). 1982;76(3):245-250.

17. Martin-Santos R, et al. Acute effects of a single, oral dose of d9-tetrahydrocannabinol (THC) and cannabidiol (CBD) administration in healthy volunteers. Curr Pharm Des. 2012;18(32):4966-4979.

18. Fusar-Poli P, et al. Distinct effects of \{delta\} 9-tetrahydrocannabinol and cannabidiol on neural activation during emotional processing. Arch Gen Psychiatry. 2009;66(1):95-105.

19. Bergamaschi MM, et al. Cannabidiol reduces the anxiety induced by simulated public speaking in treatment-naïve social phobia patients. Neuropsychopharmacology. 2011;36(6):1219-1226.

20. Sidney S. Cardiovascular consequences of marijuana use. J Clin Pharmacol. 2002;42(11 Suppl):64S-70S.

21. Pertwee RG. The diverse CB1 and CB2 receptor pharmacology of three plant cannabinoids: delta9-tetrahydrocannabinol, cannabidiol and delta9-tetrahydrocannabivarin. Br J Pharmacol. 2008;153(2):199-215.

22. McPartland JM, Duncan M, Di Marzo V, Pertwee RG. Are cannabidiol and $\Delta(9)$-tetrahydrocannabivarin negative modulators of the endocannabinoid system? A systematic review. Br J Pharmacol. 2015;172(3):737-753.

23. Penumarti A, Abdel-Rahman AA. The novel endocannabinoid receptor GPR18 is expressed in the rostral ventrolateral medulla and exerts tonic restraining influence on blood pressure. J Pharmacol Exp Ther. 2014;349(1):29-38.

24. McHugh D, Page J, Dunn E, Bradshaw HB. $\Delta(9)$-Tetrahydrocannabinol and N-arachidonyl glycine are full agonists at GPR18 receptors and induce migration in human endometrial HEC-1B cells. Br J Pharmacol. 2012;165(8):2414-2424.

25. Schwartz CE, Durocher JJ, Carter JR. Neurovascular responses to mental stress in prehypertensive humans. J Appl Physiol. 2011;110(1):76-82.

26. Barcroft H, Brod J, Hej1 BZ, Hirsjarvi EA, Kitchin AH. The mechanism of the vasodilatation in the forearm muscle during stress (mental arithmetic). Clin Sci. 1960;19:577-586.

27. Lind AR, Taylor SH, Humphreys PW, Kennelly BM, Donald KW. THE CIRCULATIORY EFFECTS OF SUSTAINED VOLUNTARY MUSCLE CONTRACTION. Clin Sci. 1964;27:229-244.

28. Delius W, Hagbarth KE, Hongell A, Wallin BG. Manoeuvres affecting sympathetic outflow in human muscle nerves. Acta Physiol Scand. 1972;84(1):82-94. 
29. Sander M, Macefield VG, Henderson LA. Cortical and brain stem changes in neural activity during static handgrip and postexercise ischemia in humans. $J$ Appl Physiol. 2010;108(6):1691-1700.

30. Ishii K, et al. Differential contribution of ACh-muscarinic and $\beta$-adrenergic receptors to vasodilatation in noncontracting muscle during voluntary one-legged exercise. Physiol Rep. 2014;2(11):e12202.

31. Hudson BD, Hébert TE, Kelly ME. Physical and functional interaction between CB1 cannabinoid receptors and beta2-adrenoceptors. Br J Pharmacol. 2010;160(3):627-642.

32. Victor RG, Leimbach WN, Seals DR, Wallin BG, Mark AL. Effects of the cold pressor test on muscle sympathetic nerve activity in humans. Hypertension. 1987;9(5):429-436.

33. Mathias CJ, Bannister R. Investigation of autonomic disorders. In: Bannister R, Mathias CJ, eds. Autonomic Failure. A textbook of clinical disorders of the autonomic nervous system. Oxford:Oxford University Press;1992:255-290.

34. Yamamoto K, Iwase S, Mano T. Responses of muscle sympathetic nerve activity and cardiac output to the cold pressor test. Jpn J Physiol. 1992;42(2):239-252.

35. Russo EB. Cannabinoids in the management of difficult to treat pain. Ther Clin Risk Manag. 2008;4(1):245-259.

36. O'Sullivan SE, Bell C. Training reduces autonomic cardiovascular responses to both exercise-dependent and -independent stimuli in humans. Auton Neurosci. 2001;91(1-2):76-84.

37. Tzadok M, et al. CBD-enriched medical cannabis for intractable pediatric epilepsy: The current Israeli experience. Seizure. 2016;35:41-44.

38. Fusar-Poli P, et al. Modulation of effective connectivity during emotional processing by Delta 9-tetrahydrocannabinol and cannabidiol. Int J Neuropsychopharmacol. 2010;13(4):421-432.

39. O'Connell BK, Gloss D, Devinsk O. Cannabinoids in treatment-resistant epilepsy: A review [published online ahead of print February 8, 2017]. Epilepsy Behav. https://doi.org/10.1016/j.yebeh.2016.11.012

40. Schutte AE, Huisman HW, van Rooyen JM, Malan NT, Schutte R. Validation of the Finometer device for measurement of blood pressure in black women. J Hum Hypertens. 2004;18(2):79-84.

41. Johnson JM, Taylor WF, Shepherd AP, Park MK. Laser-Doppler measurement of skin blood flow: comparison with plethysmography. J Appl Physiol Respir Environ Exerc Physiol. 1984;56(3):798-803. 